\title{
Entropy and canonical ensemble of hybrid quantum classical systems
}

\author{
J. L. Alonso $\odot,{ }^{1,2,3}$ C. Bouthelier $\odot,{ }^{1,2}$ A. Castro $\oplus^{1,2,4}$ J. Clemente-Gallardo $\odot,,^{1,2,3}$ and J. A. Jover-Galtier $\circledast^{1,2,5}$ \\ ${ }^{1}$ Departamento de Física Teórica, Universidad de Zaragoza, Campus San Francisco, 50009 Zaragoza, Spain \\ ${ }^{2}$ Instituto de Biocomputación y Física de Sistemas Complejos (BIFI), Universidad de Zaragoza, Edificio I+D, Mariano Esquillor s/n, \\ 50018 Zaragoza, Spain \\ ${ }^{3}$ Centro de Astropartículas y Física de Altas Energas (CAPA), Departamento de Física Teórica, \\ Universidad de Zaragoza, Zaragoza 50009, Spain \\ ${ }^{4}$ Fundación ARAID, Av. de Ranillas 1-D, planta 2, oficina B, 50018 Zaragoza, Spain \\ ${ }^{5}$ Centro Universitario de la Defensa de Zaragoza, Academia General Militar, 50090 Zaragoza, Spain
}

(Received 7 April 2020; revised 14 April 2020; accepted 16 September 2020; published 14 October 2020)

\begin{abstract}
In this work we generalize and combine Gibbs and von Neumann approaches to build, for the first time, a rigorous definition of entropy for hybrid quantum-classical systems. The resulting function coincides with the two cases above when the suitable limits are considered. Then, we apply the MaxEnt principle for this hybrid entropy function and obtain the natural candidate for the hybrid canonical ensemble (HCE). We prove that the suitable classical and quantum limits of the HCE coincide with the usual classical and quantum canonical ensembles since the whole scheme admits both limits, thus showing that the MaxEnt principle is applicable and consistent for hybrid systems.
\end{abstract}

DOI: 10.1103/PhysRevE.102.042118

\section{INTRODUCTION}

Hybrid quantum-classical (QC) systems are the natural approximation to those quantum systems containing some degrees of freedom that can be well approximated as classical variables. This possibility arises when there are two different energy or mass scales, as it happens, for instance, in molecular and condensed matter systems where the nuclei are heavy and slow, while the electrons are light and fast. Hybrid models have also been proposed to explain the measurement process $[1,2]$ : the measurement device is modeled as a classical system coupled to the quantum system to be measured. In field theory, hybrid quantum-classical systems have also been considered as candidates to describe quantum matter fields interacting with a (classical) gravitational field, as a semiclassical approximation or even a fundamental theory (see Refs. [3,4]).

The correct mathematical formalism for the dynamics and statistics of these hybrid models is not obvious. Two different points of view can be taken. On the one hand, a practical one: the construction of a hybrid theory that approximates, as closely as possible, the full quantum dynamics of the problem. Such methods can be applied to a very large array of problems in condensed matter and molecular physics and chemistry, as nonadiabatic processes play a fundamental role [5-9]. On the other hand, a fundamental, theoretical point of view: the construction of a mathematically and physically consistent theory for hybrid systems, according to different demands of consistency [1,10-27], independently of how well it may approximate the full quantum dynamics. This second approach is compulsory when the full quantum dynamics is not known, as in the case of a system of quantum matter fields interacting with gravity. In any case, it is not clear what is the best possible dynamics from any of those two points of view. Here, we assume the second one, and add to the discussion on the construction of a mathematically consistent and physically motivated hybrid theory.

The focus of this work is on the statistical mechanics of hybrid systems, regardless of the dynamics chosen for their description. In particular, we consider two open questions: first, what is the correct definition of the entropy of a hybrid system? And then, given this definition, can we use the MaxEnt formalism and obtain the canonical ensemble of a hybrid system, as we do for classical or quantum ones? Apparently, these are purely fundamental questions, but their answers are crucial for many applications, in particular, for the $a b$ initio modeling of molecules and materials and their numerical simulation methods at finite temperature (for example, see Refs. [28-33]). We determine, in a simple way, the equilibrium ensemble that the numerical methods must reproduce and the entropy function they must consider.

The structure of the paper is as follows. In Sec. II we will first discuss the proper definition of the hybrid entropy function. Then, in Sec. III we will derive the hybrid canonical ensemble (HCE) as the one that maximizes this entropy, subject to the constraint of a given expectation value for the energy (MaxEnt principle). The resulting ensemble had been perhaps implicitly assumed before, but few times explicitly spelled, and never, to our knowledge, derived from the general principle of entropy maximization. We will also briefly discuss some relevant properties of the resulting ensemble.

Finally, in Sec. IV we will summarize our main conclusions.

\section{THE ENTROPY OF A HYBRID QC SYSTEM}

A correct statistical mechanical definition of any system departs from the definition of a sample space: a set of 
statistically independent states, i.e., a basis of mutually exclusive events (MEE), which can be unequivocally characterized by the results of an experiment. Let us start by recalling the basic definitions in the purely classical or purely quantum cases.

In classical systems, a basis of MEEs is simply the phase space $\mathcal{M}_{C}$, the set of all positions and momenta of the classical particles: $\mathcal{M}_{C}=\left\{(Q, P) \mid Q \in \mathbb{R}^{n}, P \in \mathbb{R}^{n}\right\}$, where $n$ is the number of classical degrees of freedom. Any point in this phase space defines an exclusive event from any other event. Observables are real functions on this $\mathcal{M}_{C}$. Statistical mechanics for classical systems can then be described by using ensembles on this phase space, i.e., (generalized) ${ }^{1}$ probability distribution functions (PDFs) $F_{\mathrm{C}}$

In quantum systems, the states are rays of a Hilbert space $\mathcal{H}$, i.e., the analogous to the classical phase space is the projective space, $\mathcal{M}_{Q}=\mathcal{P} \mathcal{H}$. We will represent its points as the projectors on 1-dimensional subspaces of the Hilbert space $\hat{\rho}_{\psi}=\frac{|\psi\rangle\langle\psi|}{\langle\psi \mid \psi\rangle}$, with $|\psi\rangle \in \mathcal{H} \backslash\{\overrightarrow{0}\}$. Even though all of the states in $\mathcal{M}_{Q}$ are physically legitimate, they are not mutually exclusive. Indeed, if the system has been measured to be, with probability one, in a state $\hat{\rho}_{\psi_{1}}$, the probability of measuring it to be in other state $\hat{\rho}_{\psi_{2}}$ is not zero, unless they are orthogonal: $\hat{\rho}_{\psi_{1}}, \hat{\rho}_{\psi_{2}}$ are MEE only if $\left\langle\psi_{1} \mid \psi_{2}\right\rangle=0$. As a consequence, considering generalized probability density functions $F_{\mathrm{Q}}$ over the Hilbert space (or over the projective space of rays) to define ensembles, following the classical analogy, results in overcounting the same outcome for a hypothetical experiment in a nontrivial way. One way to see this clearly is that many different $F_{\mathrm{Q}}$ can correspond to exactly the same ensemble (i.e., they are physically indistinguishable). The correct way to get a sample space of MEEs is therefore considering a basis of orthogonal events. From this idea, von Neumann [34] derived the density matrix formalism, which contains all the physically relevant statistically nonredundant information in a compact way. A density matrix can be obtained from a PDF $F_{\mathrm{Q}}$ in the quantum state space as

$$
\hat{\rho}\left[F_{\mathrm{Q}}\right]=\int \mathrm{d} \mu_{Q}\left(\hat{\rho}_{\psi}\right) F_{\mathrm{Q}}\left(\hat{\rho}_{\psi}\right) \hat{\rho}_{\psi},
$$

where we represent by $d \mu_{Q}$ the volume element on $\mathcal{M}_{Q}$. Analogously, in the following, we will represent by $d \mu_{C}$ the volume element on $\mathcal{M}_{C}$.

We move on now to QC theories. Despite the various proposals referenced above, one can perhaps establish a common denominator. The classical part is described by a set of position $Q \in \mathbb{R}^{n}$ and momenta $P \in \mathbb{R}^{n}$ variables, that we will hereafter collectively group as $\xi=(Q, P)$. The quantum part is described by a complex Hilbert space $\mathcal{H}$. Observables are Hermitian operators on $\mathcal{H}$, and they may depend parametrically on the classical variables, $\hat{A}(\xi): \mathcal{H} \rightarrow$ $\mathcal{H}$. Those observables defined on the classical subsystem are just $\xi$-functions times the identity, i.e., $\hat{A}(\xi)=A(\xi) \hat{I}$; those observables defined on the quantum subsystem only are operators that lack the $\xi$-dependence.

\footnotetext{
${ }^{1}$ We introduce the adjective generalized to refer to the set of generalized functions (or distributions) and include, for instance, Dirac $\delta$ functions.
}

We are going to consider two different approaches to the definition of the entropy, one based on the usual approach to classical systems, and another one inspired by the quantum case.

\section{A. A Gibbs-entropy for hybrid systems?}

The formal similarities of one of the best known hybrid dynamical models, Ehrenfest dynamics, with the classical one (see Refs. [18,27] for details) may lead to consider hybrid systems as formally closer to classical than to quantum dynamics. Indeed, Ehrenfest dynamics can be given a Hamiltonian structure (see Refs. [27,35]) in terms of

(1) a Hamiltonian function constructed as

$$
f_{H}\left(\xi, \hat{\rho}_{\psi}\right)=\operatorname{Tr}\left(\hat{H}(\xi) \hat{\rho}_{\psi}\right)=\frac{\langle\psi \mid \hat{H}(\xi) \psi\rangle}{\langle\psi \mid \psi\rangle},
$$

(2) and a Poisson bracket obtained as the combination of the Poisson bracket of classical mechanics and the canonical Poisson bracket of quantum systems (see Refs. [36,37]).

This fact makes Ehrenfest dynamical description of hybrid system formally analogous to a classical Hamiltonian dynamical system. When considering the definition of hybrid statistical systems, we can then consider a hybrid (generalized) PDF $F_{\mathrm{H}}$ defined over the hybrid phase space $\mathcal{M}_{H}=$ $\mathcal{M}_{C} \times \mathcal{M}_{Q}$, in an analogous manner to the definition of classical statistical systems. The Hamiltonian nature of the dynamics allows to define a Liouville equation for $F_{\mathrm{H}}$ in a straightforward manner (see Refs. [18,27]).

Within that framework, it is also tempting to borrow the notion of entropy from classical statistical mechanics and define a Gibbs-like function associated with the density function $F_{\mathrm{H}}$ in the following form:

$$
S_{\mathrm{G}}\left[F_{\mathrm{H}}\right]=-k_{B} \int_{\mathcal{M}_{H}} d \mu_{H}\left(\xi, \rho_{\psi}\right) F_{\mathrm{H}}\left(\xi, \rho_{\psi}\right) \log \left[F_{\mathrm{H}}\left(\xi, \rho_{\psi}\right)\right],
$$

where $k_{B}$ represents the Boltzman costant and $d \mu_{H}$ represents the volume element on $\mathcal{M}_{H}$ which can be written in terms of the classical and quantum volume elements as $d \mu_{H}=d \mu_{C} \wedge$ $d \mu_{Q}$.

Notice that this entropy function is well defined for classical systems, where the points of phase-space correspond to mutually exclusive events. Therefore, when considering $S_{\mathrm{G}}$ we are adding all points of the phase space $\mathcal{M}_{H}$ as if they were mutually exclusive. Thus, we treat them as classical statistical systems, where being at a given point in phase space excludes the possibility of being at a different point. Hence, we are not weighting correctly the quantum subsystems from the physical point of view, ruining the function ability to measure physical information for the hybrid system.

Despite this fact, this entropy function has been implicitly assumed several times when considering hybrid or even purely quantum statistical systems (see Refs. [27,38-40]), when defining the so called Schrödinger-Gibbs (SG) ensemble or the corresponding Schrödinger microcanonical ensemble. Thus, SG represents a canonical ensemble where the probability density is written by assigning to each state the Gibbs weight associated with the expectation value of the Hamiltonian, instead of the operator itself. But the bad physical 
properties of $S_{\mathrm{G}}$ lead to very strange and un-physical properties for the corresponding thermodynamic functions. In particular, this was the case when the Schrödinger-Gibbs ensemble was analyzed in Ref. [41]. Nonetheless, notice that $S_{\mathrm{G}}$ is a mathematically consistent entropy function, despite the unphysical properties of the Statistical Mechanics it defines.

\section{B. Gibbs-von Neumann entropy}

From our analysis above, it is clear that the straightforward extension of Gibbs classical entropy function to hybrid systems leads to inconsistencies because the points of hybrid phase space do not define mutually exclusive events as the classical phase space points do. To do statistical mechanics in a consistent way with the nature of its quantum subsystem, one must reconsider the notion of mutually exclusive events, and combine the classical and the quantum notions of MEE. The combined hybrid phase space is now $\mathcal{M}_{H}=$ $\mathcal{M}_{C} \times \mathcal{M}_{Q}$. But, we must consider that two hybrid states $\left(\xi_{1}, \hat{\rho}_{\psi_{1}}\right),\left(\xi_{2}, \hat{\rho}_{\psi_{2}}\right) \in \mathcal{M}_{H}$ represent MEEs if and only if $\xi_{1} \neq$ $\xi_{2}$ or $\left\langle\psi_{1} \mid \psi_{2}\right\rangle=0$.

The next step is to define a probability distribution on the set of MEEs of $\mathcal{M}_{H}$. Following von Neumann idea and the mathematical construction of Gleason theorem [42], we can build a hybrid density matrix to represent the hybrid probability in a consistent way. As the physical properties of the hybrid system, in general, combine the states of $\mathcal{M}_{C}$ and $\mathcal{M}_{Q}$ (for instance, the total energy of the system), we cannot expect both sets to be independent from the probabilistic point of view. Nonetheless, we can assume that we can simultaneously measure any classical observable and any hybrid observable of the form $\hat{A}(\xi)$. This fact permits to define the conditional probabilities $p(a \mid \xi)$ : the probability of measuring an eigenvalue a of operator $\hat{A}(\xi)$, given that the classical subsystem is at state $\xi \in \mathcal{M}_{C}$. The probabilities associated to the hybrid measurement can then be decomposed into the marginal probability associated to the classical phase space, $F_{C}(\xi)$, and the conditional probabilities associated to the measurement of $\hat{A}(\xi)$, given $\xi$ :

$$
p(a, \xi)=F_{\mathrm{C}}(\xi) p(a \mid \xi)
$$

For these quantum conditional probabilities $p(a \mid \xi)$, all the requirements of Gleason's theorem [42] apply, and one may therefore define, at each $\xi$ point, a density matrix $\hat{\rho}^{\xi}$. It provides the probabilities of measuring an eigenvalue $a$ of observable $\hat{A}(\xi)$, given $\xi$, through the usual Born rule: $p(a \mid \xi)=\operatorname{Tr}\left[\hat{\rho}^{\xi} \hat{\pi}_{a}(\xi)\right]$, where $\hat{\pi}_{a}(\xi)$ is the projector onto the eigen-subspace associated to $a$. From this, we can define the hybrid density matrix as the $\xi$-dependent matrix:

$$
\hat{\rho}(\xi)=F_{\mathrm{C}}(\xi) \hat{\rho}^{\xi}
$$

such that $p(a, \xi)=\operatorname{Tr}[\hat{\rho}(\xi) \hat{\pi}(a)]$. Notice that, strictly speaking, Gleason theorem ensures the existence and uniqueness of the density matrix $\hat{\rho}^{\xi}$ only for Hilbert spaces of dimension at least 3 . However, the recent developments based on positive-operator-valued measures (POVM) (see, for instance, Refs. $[43,44])$ allow to prove a more general formulation of Gleason theorem for quantum states which is valid in dimension 2 , but in that case the construction is not based on orthogonality of the rank-one projectors but on a more global set of effects.

In conclusion, the probability distribution on the set of MEEs of hybrid states can be written as a family of quantum density operators parameterized by the classical degrees of freedom, $\hat{\rho}(\xi)$. For each $\xi, \hat{\rho}(\xi)$ is a self-adjoint and nonnegative operator, which is normalized on the full hybrid sample space:

$$
\int_{\mathcal{M}_{C}} \mathrm{~d} \mu_{C}(\xi) \operatorname{Tr}[\hat{\rho}(\xi)]=1
$$

This is an immediate consequence of the normalization of $F_{\mathrm{C}}(\xi)=\operatorname{Tr} \hat{\rho}(\xi)\left[\int_{\mathcal{M}_{C}} d \mu_{C}(\xi) F_{\mathrm{C}}(\xi)=1\right]$ and of $\hat{\rho}^{\xi}\left(\operatorname{Tr} \hat{\rho}^{\xi}=\right.$ $1)$. Given a hybrid state determined by the classical point $\xi$ [which has probability $\operatorname{Tr} \hat{\rho}(\xi)$ ], and a quantum state represented by the projector $\hat{\pi}$, the probability of measuring the system to be in that state is given by $\operatorname{Tr}[\hat{\rho}(\xi) \hat{\pi}]$. These $\xi$ dependent density matrices have already been used before, for example, by Aleksandrov [25], or obtained by taking the partial classical limit in the Wigner transformation of the full quantum density matrix, in the quantum-classical Liouville equation method [26].

Let us consider now how to define the entropy of these hybrid states. For any bivariate distribution $p(x, y)$ of two sets of random variables $(X, Y)$, the entropy $S(p)$ decomposes as

$$
S(p)=S\left(p_{X}\right)+\sum_{x} p_{X}(x) S\left(p_{Y \mid x}\right),
$$

where $p_{X}(x)=\sum_{y} p(x, y)$ is the marginal distribution of $X$, and $p_{Y \mid x}$ is the conditional probability of $Y$ given $x$. This general result must be applicable to the decomposition Eqs. (4) and (5). Therefore, the entropy of the hybrid system must be equal to the sum of the (classical) entropy $\left(S_{C}\right)$ of the marginal classical distribution $F_{\mathrm{C}}(\xi)$ and the average, over $F_{\mathrm{C}}(\xi)$, of the (von Neumann) entropy associated to the conditional probability $\rho^{\xi}$, i.e.,

$$
\begin{aligned}
S[\hat{\rho}(\xi)]= & \overbrace{-k_{B} \int_{\mathcal{M}_{C}} d \mu_{C}(\xi) F_{\mathrm{C}}(\xi) \log \left(F_{\mathrm{C}}(\xi)\right)}^{S_{C}\left(F_{\mathrm{C}}\right)} \\
& +\int_{\mathcal{M}_{C}} d \mu_{C}(\xi) F_{\mathrm{C}}(\xi) \underbrace{\left[-k_{B} \operatorname{Tr}\left(\hat{\rho}^{\xi} \log \hat{\rho}^{\xi}\right)\right]}_{S_{\mathrm{vN}}\left(\hat{\rho}^{\xi}\right)}
\end{aligned}
$$

It is immediate then to rewrite this as

$$
S[\hat{\rho}(\xi)]=-k_{B} \int_{\mathcal{M}_{C}} \mathrm{~d} \mu_{C}(\xi) \operatorname{Tr}[\hat{\rho}(\xi) \log \hat{\rho}(\xi)],
$$

which is our proposal for the hybrid QC entropy. To the best of our knowledge, this is the first rigorous proposal of an entropy function for a hybrid quantum-classical system. If the classical subsystem is pure [i.e., $F_{\mathrm{C}}(\xi)=\delta\left(\xi-\xi_{0}\right)$ ], then the classical entropy vanishes and the entropy above reduces to von Neumann entropy. Analogously, when the quantum state is pure and independent of the classical state, the von Neumann entropy of $\rho^{\xi}$ vanishes, and the expression above reduces to the classical entropy function. Therefore, the entropy function Eq. (9) combines the classical and quantum information in a consistent way, and has the correct classical and quantum limits. 


\section{THE MAXENT PRINCIPLE FOR HYBRID QC SYSTEMS}

\section{A. MaxEnt principle for the hybrid entropy function}

The maximum entropy principle is one of the standard procedures to derive the canonical ensemble at both the classical or the quantum level. First, one must assume that the system is in equilibrium. Then, one can find the canonical ensemble as the solution of the MaxEnt problem: given a certain thermodynamic system and an entropy function $S$, find the equilibrium ensemble which maximizes $S$ among those with a fixed value of the average energy $E=\langle\hat{H}(\xi)\rangle$.

In the following, we will prove that the canonical ensemble that results of this maximization, for the hybrid case, is given by

$$
\begin{gathered}
\hat{\rho}_{\mathrm{HCE}}(\xi)=\frac{e^{-\beta \hat{H}(\xi)}}{Z_{\mathrm{HCE}}(\beta)}, \\
Z_{\mathrm{HCE}}(\beta)=\int_{\mathcal{M}_{C}} \mathrm{~d} \mu_{C}(\xi) \operatorname{Tr}\left(e^{-\beta \hat{H}(\xi)}\right),
\end{gathered}
$$

where $\hat{H}(\xi)$ is the Hamiltonian [typically decomposed into a classical and a quantum part, as $\left.f_{H}^{c}(\xi) \hat{I}+\hat{H}_{Q}(\xi)\right], Z_{\mathrm{HCE}}(\beta)$ is the partition function, and $\beta$ is a constant, determined by the choice of $E$, that is used to define the (inverse of the) temperature. Note that this ensemble had been perhaps implicitly assumed before, but seldom explicitly written [45] and, to our knowledge, never derived. Notice that the orthogonal projectors of its spectral decomposition coincide with those of the adiabatic basis.

The problem can be addressed as a constrained optimization problem: find the density matrix that maximizes $S$ in Eq. (9), subject to the following constraints:

$$
\begin{array}{r}
C_{N}[\hat{\rho}(\xi)]:=\int_{\mathcal{M}_{C}} \mathrm{~d} \mu_{C}(\xi) \operatorname{Tr}[\hat{\rho}(\xi)]-1=0, \\
C_{E}[\hat{\rho}(\xi)]:=\int_{\mathcal{M}_{C}} \mathrm{~d} \mu_{C}(\xi) \operatorname{Tr}[\hat{\rho}(\xi) \hat{H}(\xi)]-E=0 .
\end{array}
$$

These can be incorporated via Lagrange multipliers, defining the full optimization functional to be

$$
\mathcal{S}:=S-\lambda_{N} C_{N}-\lambda_{E} C_{E} .
$$

Without loss of generality, let us work in the ( $\xi$-dependent) basis of eigenstates of the Hamiltonian (the adiabatic basis). First, we will consider the optimization over a reduced set of density matrices: those which are diagonal in this adiabatic basis. The terms in Eq. (14) then read

$$
\begin{gathered}
S\left[\left\{\rho_{i i}\right\}\right]=-k_{B} \int_{\mathcal{M}_{C}} d \mu_{C}(\xi) \sum_{i} \rho_{i i}(\xi) \log \left(\rho_{i i}(\xi),\right. \\
C_{N}\left[\left\{\rho_{i i}\right\}\right]=\int_{\mathcal{M}_{C}} d \mu_{C}(\xi) \sum_{i} \rho_{i i}(\xi)-1, \\
C_{E}\left[\left\{\rho_{i i}\right\}\right]=\int_{\mathcal{M}_{C}} d \mu_{C}(\xi) \sum_{i} H_{i}(\xi) \rho_{i i}(\xi)-E .
\end{gathered}
$$

Taking derivatives and setting them to zero leads immediately to

$$
\rho_{i i}(\xi)=Z_{\mathrm{HCE}}(\beta)^{-1} e^{-\beta H_{i}(\xi)},
$$

where $\beta=\frac{\lambda_{E}}{k_{B}}$.
We consider now a general density matrix $\hat{\tilde{\rho}}(\xi)$, whose nondiagonal elements may be nonzero, fulfilling the two constraint Eqs. (12) and (13). Since it is Hermitian with nonnegative eigenvalues, it satisfies Klein's lemma [46]:

$$
-\operatorname{Tr}\left[\hat{\tilde{\rho}}(\xi) \log (\hat{\tilde{\rho}}(\xi)] \leqslant-\sum_{i} \tilde{\rho}_{i i}(\xi) \log \left[\tilde{\rho}_{i i}(\xi)\right],\right.
$$

where $\tilde{\rho}_{i i}(\xi)$ are its diagonal elements (the equality only holds if it is actually diagonal). As the constraint Eqs. (12) and (13) in the adiabatic basis only depend on the diagonal elements of $\hat{\rho}(\xi)$, we may conclude that for any nondiagonal density matrix that fulfills the constraints there exists a diagonal one (defined to be the one whose diagonal entries are the same) that also fulfills the constraints and has a larger entropy. The global maximum, therefore, has to be found among the diagonal ones, and is the one given in Eq. (18). This concludes the proof.

\section{B. Properties of the HCE}

Let us now check that the ensemble thus defined fulfills some very natural requirements:

(1) Additivity. If two systems are in the canonical ensemble equilibrium at the same temperature, then they must also be at equilibrium when we consider them to form a single system with two (independent) subsystems. Extensive variables as the energy and entropy must be additive.

This can be proven for the HCE in the following way. If $\hat{H}_{1}\left(\xi_{1}\right)$ and $\hat{H}_{2}\left(\xi_{2}\right)$ are the Hamiltonians of both systems, then the combined one is

$$
\hat{H}(\xi)=\hat{H}_{1}\left(\xi_{1}\right) \otimes \hat{\mathbb{I}}_{2}+\hat{\mathbb{I}}_{1} \otimes \hat{H}_{2}\left(\xi_{2}\right),
$$

where $\xi=\left(\xi_{1}, \xi_{2}\right)$.

As the two terms of Eq. (20) trivially commute,

$$
e^{-\beta \hat{H}(\xi)}=e^{-\beta \hat{H}_{1}\left(\xi_{1}\right)} \otimes e^{-\beta \hat{H}_{2}\left(\xi_{2}\right)},
$$

and because of this,

$$
\begin{aligned}
& \int_{\mathcal{M}_{C_{1}} \times \mathcal{M}_{C_{2}}} d \mu_{C}\left(\xi_{1}, \xi_{2}\right) \operatorname{Tr} e^{-\beta \hat{H}(\xi)} \\
& \quad=\int_{\mathcal{M}_{C_{1}}} d \mu_{C}\left(\xi_{1}\right) \operatorname{Tr} e^{-\beta \hat{H}_{1}\left(\xi_{1}\right)} \int_{\mathcal{M}_{C_{2}}} d \mu_{C}\left(\xi_{2}\right) \operatorname{Tr} e^{-\beta \hat{H}_{2}\left(\xi_{2}\right)} .
\end{aligned}
$$

Thus, we can just write

$$
\hat{\rho}(\xi)=\hat{\rho}_{1}\left(\xi_{1}\right) \otimes \hat{\rho}_{2}\left(\xi_{2}\right) .
$$

This factorization of $\hat{\rho}(\xi)$ immediately implies the additivity of the internal energy Eq. (13) and of the entropy Eq. (9).

(2) The classical canonical ensemble, which maximizes Gibbs entropy, is recovered when only one quantum energy state exists.

(3) The quantum canonical ensemble, which maximizes von Neumann entropy, is recovered when only one classical point is allowed.

(4) If the QC coupling is turned off (the quantum Hamiltonian $\hat{H}_{Q}$ is independent of the classical variables and vice versa), then the HCE becomes the product of the classical and quantum canonical ensembles, which maximize the sum of their respective entropies independently. 


\section{Dynamics}

Another extra condition that an equilibrium ensemble must obviously verify is missing in the previous list: stationarity under the dynamics of the microstates. However, up to now we have disregarded the dynamics, and derived the canonical ensemble from very broad assumptions, freed from dynamical arguments. The dynamics is neither relevant for the definition of the entropy function nor affects directly the solution of the MaxEnt condition. For instance, $\mathrm{MaxS}_{\mathrm{G}}$ defines the MaxEnt solution for the entropy function $S_{\mathrm{G}}$ [Eq. (3)], independently of the dynamics of the microstates we consider. The existence of dynamics having it as an equilibrium point would be an extra requirement for the definition of a thermodynamical ensemble.

On the other hand, we also proved above that the MaxEnt solution of the true hybrid entropy function Eq. (9) is the HCE. This implies that the only possible ensemble which can be considered to represent the canonical ensemble of a hybrid system is the HCE. Is there a dynamics that makes it also stationary? Trivially, the commutator with $\hat{H}(\xi)$ (i.e., a generalized von Neumann equation) does, but many others may also be possible. We will analyze this issue in a forthcoming publication.

\section{CONCLUSIONS}

It has been the purpose of this paper to shed some light into the issue of the entropy and the canonical equilibrium expression for hybrid systems. We have first discussed the definition for the entropy of an ensemble of hybrid systems.
We have done it by making very general assumptions on the hybrid theory, but without any consideration for the particular dynamics. We have considered two different alternatives, one based on probability densities on the hybrid phase space and another based on projectors and the notion of hybrid mutually exclusive events. The first case leads to a Gibbs-like function which treats the hybrid system as a direct analog of a classical system. We have shown how that entropy function assigns the wrong weight to hybrid events and because of this fails to produce a physically meaningful Thermodynamics. The second proposal departs from the information-theory definition of entropy, and carefully considers the principle of mutually exclusive events. The resulting hybrid entropy function weights correctly the hybrid exclusive events and defines a physically consistent thermodynamical entropy.

Then, we have derived the HCE as the one that fulfills the MaxEnt principle with respect to the hybrid entropy function, using it for the first time for hybrid quantum-classical systems. Furthermore, we verified that the HCE reproduces the classical and quantum cases when the suitable limits are considered. Hence, we can claim that the MaxEnt principle is applicable and consistent for hybrid quantum-classical systems.

\section{ACKNOWLEDGMENTS}

The authors would like to thank Prof. Floria and Prof. Zueco for their very useful suggestions. Partial financial support by MINECO Grant No. FIS2017-82426-P is acknowledged. C.B. acknowledges financial support by Gobierno de Aragón through the grant defined in ORDEN IIU/1408/2018.
[1] L. Diósi, Hybrid quantum-classical master equations, Phys. Scr. T 163, 014004 (2014).

[2] N. Buric, D. B. Popovic, M. Radonjic, and S. Prvanovic, Hybrid quantum-classical model of quantum measurements, Phys. Rev. A 87, 054101 (2013).

[3] P. Martin-Dussaud and C. Rovelli, Evaporating black-to-white hole, Classical Quantum Gravity 36, 245002 (2019).

[4] A. Tilloy, Does gravity have to be quantized? Lessons from nonrelativistic toy models, J. Phys.: Conf. Ser. 1275, 012006 (2019).

[5] J. C. Tully, Mixed quantum-classical dynamics, Faraday Discuss. 110, 407 (1998).

[6] T. Yonehara, K. Hanasaki, and K. Takatsuka, Fundamental approaches to nonadiabaticity: Toward a chemical theory beyond the Born-Oppenheimer paradigm, Chem. Rev. 112, 499 (2012).

[7] I. Tavernelli, Nonadiabatic molecular dynamics simulations: Synergies between theory and experiments, Acc. Chem. Res. 48, 792 (2015).

[8] R. Crespo-Otero and M. Barbatti, Recent advances and perspectives on nonadiabatic mixed quantum-classical dynamics, Chem. Rev. 118, 7026 (2018).

[9] B. F. E. Curchod and T. J. Martínez, Ab initio nonadiabatic quantum molecular dynamics, Chem. Rev. 118, 3305 (2018).

[10] O. V. Prezhdo and V. V. Kisil, Mixing quantum and classical mechanics, Phys. Rev. A 56, 162 (1997).
[11] V. V. Kisil, A quantum-classical bracket from $p$-mechanics, Europhys. Lett. 72, 873 (2005).

[12] O. V. Prezhdo, A quantum-classical bracket that satisfies the Jacobi identity., J. Chem. Phys. 124, 201104 (2006).

[13] L. L. Salcedo, Comment on "A quantum-classical bracket that satisfies the Jacobi identity" [J. Chem. Phys. 124, 201104 (2006)], J. Chem. Phys. 126, 057101 (2007).

[14] F. Agostini, S. Caprara, and G. Ciccotti, Do we have a consistent nonadiabatic quantum-classical mechanics?, Europhys. Lett. 78, 30001 (2007).

[15] V. V. Kisil et al., Comment on "Do we have a consistent nonadiabatic quantum-classical mechanics?" by F. Agostini, Europhys. Lett. 89, 50005 (2010).

[16] F. Agostini, S. Caprara, and G. Ciccotti, Reply to the Comment by V. V. Kisil, Europhys. Lett. 89, 50006 (2010).

[17] M. J. W. Hall, Consistent classical and quantum mixed dynamics, Phys. Rev. A 78, 042104 (2008).

[18] N. Buri, D. B. Popovi, M. Radonji, and S. Prvanovi, Hamiltonian formulation of statistical ensembles and mixed states of quantum and hybrid systems, Found. Phys. 43, 1459 (2013).

[19] A. Peres and D. R. Terno, Hybrid classical-quantum dynamics, Phys. Rev. A 63, 022101 (2001).

[20] D. R. Terno, Inconsistency of quantum-classical dynamics, and what it implies, Found. Phys. 36, 102 (2006).

[21] L. L. Salcedo, Absence of classical and quantum mixing, Phys. Rev A 54, 3657 (1996). 
[22] V. Gil and L. L. Salcedo, Canonical bracket in quantumclassical hybrid systems, Phys. Rev. A 95, 012137 (2017).

[23] J. Caro and L. L. Salcedo, Impediments to mixing classical and quantum dynamics, Phys. Rev. A 60, 842 (1999).

[24] H. Elze, Linear dynamics of quantum-classical hybrids, Phys. Rev. A 85, 052109 (2012).

[25] I. V. Aleksandrov, The statistical dynamics of a system consisting of a classical and a quantum system, Z. Naturforsch 36a, 902 (1981).

[26] R. Kapral and G. Ciccotti, Mixed quantum-classical dynamics, J. Chem. Phys. 110, 8919 (1999).

[27] J. L. Alonso, A. Castro, J. Clemente-Gallardo, J. C. Cuchí, P. Echenique, and F. Falceto, Statistics and Nosé formalism for Ehrenfest dynamics, J. Phys. A: Math. Theor. 44, 395004 (2011).

[28] A. Alavi, J. Kohanoff, M. Parrinello, and D. Frenkel, Ab Initio Molecular Dynamics with Excited Electrons, Phys. Rev. Lett. 73, 2599 (1994).

[29] M. P. Grumbach, D. Hohl, R. M. Martin, and R. Car, Ab initio molecular dynamics with a finite-temperature density functional, J. Phys.: Condens. Matter 6, 1999 (1994).

[30] P. L. Silvestrelli, A. Alavi, M. Parrinello, and D. Frenkel, $A b$ Initio Molecular Dynamics Simulation of Laser Melting of Silicon, Phys. Rev. Lett. 77, 3149 (1996).

[31] P. Ji and Y. Zhang, Femtosecond laser processing of germanium: An ab initio molecular dynamics study, J. Phys. D: Appl. Phys. 46, 495108 (2013).

[32] H. R. Rüter and R. Redmer, Ab Initio Simulations for the Ion-Ion Structure Factor of Warm Dense Aluminum, Phys. Rev. Lett. 112, 145007 (2014).

[33] V. V. Karasiev, T. Sjostrom, and S. B. Trickey, Finitetemperature orbital-free DFT molecular dynamics: Coupling Profess and Quantum Espresso, Comput. Phys. Commun. 185, 3240 (2014).

[34] J. Von Neumann, Mathematical Foundations of Quantum Mechanics (Princeton University Press, Princeton, NJ, 1955).

[35] F. A. A. Bornemann, P. Nettesheim, and C. Schütte, Quantumclassical molecular dynamics as an approximation to full quantum dynamics, J. Chem. Phys, 105, 1074 (1996).

[36] T. Kibble, Geometrization of quantum mechanics, Commun. Math. Phys. 65, 189 (1979).
[37] A. Heslot, Quantum mechanics as a classical theory, Phys. Rev. D 31, 1341 (1985).

[38] D. C. Brody and L. P. Hughston, The quantum canonical ensemble, J. Math. Phys. 39, 6502 (1998).

[39] G. Jona-Lasinio and C. Presilla, On the statistics of quantum expectations for systems in thermal equilibrium, in Quantum Mechanics: Are There Quantum Jumps? - and On the Present Status of Quantum Mechanics, edited by A. Bassi, D. Dürr, T. Weber, and N. Zanghi, AIP Conf. No. 844 (AIP, College Park, MD, 2006), pp. 200-205.

[40] M. Campisi, Quantum fluctuation relations for ensembles of wave functions, New J. Phys. 15, 115008 (2013).

[41] J. L. Alonso, A. Castro, J. Clemente-Gallardo, J. C. Cuchí, P. Echenique, J. G. Esteve, and F. Falceto, Nonextensive thermodynamic functions in the Schrödinger-Gibbs ensemble, Phys. Rev. E 91, 022137 (2015).

[42] A. M. Gleason, Measures on the closed subspaces of a Hilbert space, J. Math. Mech. 6, 885 (1957).

[43] P. Busch, Quantum States and Generalized Observables: A Simple Proof of Gleason's Theorem, Phys. Rev. Lett. 91, 120403 (2003).

[44] C. M. Caves, C. A. Fuchs, K. K. Manne, and J. M. Renes, Gleason-type derivations of the quantum probability rule for generalized measurements, Found. Phys. 34, 193 (2004).

[45] For example, it was given in Ref. [47], where it was claimed to be the partial classical limit of the fully quantum canonical ensemble. It was also presented as the zeroth order term in a classical-limit expansion of the partial Wigner transformation of the quantum canonical ensemble in Ref. [26]. Finally, in footnote 30 of Ref. [48], some of the current authors already hinted, without proof, the result demonstrated here.

[46] O. Klein, Zur quantenmechanischen Begründung des zweiten Hauptsatzes der Wärmelehre, Zeitschrift für Physik 72, 767 (1931).

[47] F. Mauri, R. Car, and E. Tosatti, Canonical statistical averages of coupled, Europhys. Lett. 24, 431 (1993).

[48] J. L. Alonso, A. Castro, J. Clemente-Gallardo, P. Echenique, J. J. Mazo, V. Polo, A. Rubio, and D. Zueco, Nonadiabatic effects within a single thermally averaged potential energy surface: Thermal expansion and reaction rates of small molecules, J. Chem. Phys. 137, 22A533 (2012). 